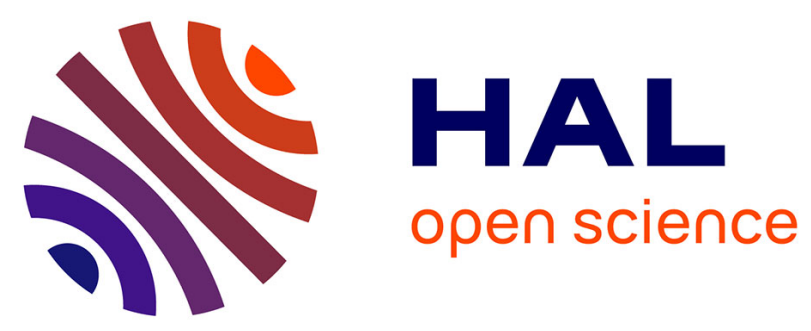

\title{
Decorated 2D quasicrystals - structure factor for clusters and average Patterson analysis
}

Janusz Wolny, Bartlomiej Kozakowski, Aleksandra Dabrowska

\section{To cite this version:}

Janusz Wolny, Bartlomiej Kozakowski, Aleksandra Dabrowska. Decorated 2D quasicrystals - structure factor for clusters and average Patterson analysis. Philosophical Magazine, 2005, 86 (03-05), pp.637643. 10.1080/14786430500254016. hal-00513571

\section{HAL Id: hal-00513571 \\ https://hal.science/hal-00513571}

Submitted on 1 Sep 2010

HAL is a multi-disciplinary open access archive for the deposit and dissemination of scientific research documents, whether they are published or not. The documents may come from teaching and research institutions in France or abroad, or from public or private research centers.
L'archive ouverte pluridisciplinaire HAL, est destinée au dépôt et à la diffusion de documents scientifiques de niveau recherche, publiés ou non, émanant des établissements d'enseignement et de recherche français ou étrangers, des laboratoires publics ou privés. 


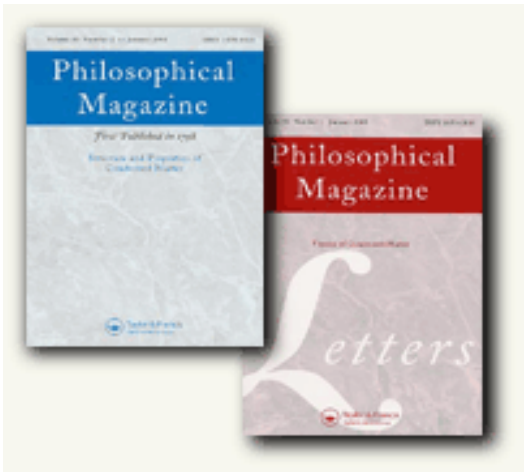

\section{Decorated 2D quasicrystals - structure factor for clusters and average Patterson analysis}

\begin{tabular}{|c|c|}
\hline Journal: & Philosophical Magazine \& Philosophical Magazine Letters \\
\hline Manuscript ID: & TPHM-05-May-0222.R1 \\
\hline Journal Selection: & Philosophical Magazine \\
\hline $\begin{array}{r}\text { Date Submitted by the } \\
\text { Author: }\end{array}$ & 07-Jul-2005 \\
\hline Complete List of Authors: & $\begin{array}{l}\text { Wolny, Janusz; AGH University of Science and Technology, Faculty } \\
\text { of Physics and Applied Computer Science } \\
\text { Kozakowski, Bartlomiej; AGH University of Science and Technology } \\
\text { Faculty of Physics and Applied Computer Science } \\
\text { Dabrowska, Aleksandra; AGH University of Science and Technology } \\
\text { Faculty of Physics and Applied Computer Science }\end{array}$ \\
\hline Keywords: & quasicrystals, clusters \\
\hline Keywords (user supplied): & Average unit cell \\
\hline
\end{tabular}

\section{S) ScholaroNE \\ Manuscript Central}




\title{
Decorated 2D quasicrystals - structure factor for clusters and average Patterson analysis
}

\author{
J. Wolny, B. Kozakowski and A. Dąbrowska, \\ Faculty of Physics and Applied Computer Science, AGH University of Science and \\ Technology, Kraków, Poland
}

PACS numbers: 61.44.Br, 61.43.-j, 61.10.Dp

Keywords: Quasicrystals, Clusters, Average unit cell

\begin{abstract}
Covering two-dimensional Penrose atomic structure with different kite-clusters consisting of four, seven and seventeen atoms is discussed. These clusters are compared to the well-known 33-atom Gummelt's cluster. The number and concentrations of different atoms decorating the clusters were found. An analytical formula for the structure factor of decorated clusters was derived in the statistical approach.
\end{abstract}

\section{Introduction}

Since the discovery of quasicrystals many methods of their structural analysis have been developed. For instance: cut and project method [1-4], average unit cell approach [5-7] or covering with clusters or rhombuses [7-10]. This paper presents a method for obtaining structure factor for Penrose lattice covered with various kinds of clusters. Especially we take into account three different kite-clusters and Gummelt's cluster. To the former we assign symbols (K4), (K7), (K17) - if a cluster contains four, seven or seventeen atoms - and to the latter symbol (G33), because Gummelt's cluster is built of 33 atoms. Kite-cluster was mentioned by Gruenbaum and Shephard [12],

In [7] we already showed the way to get the structure factor for (K17). Here we would like to extend the analysis to $(\mathrm{K} 7)$ and $(\mathrm{K} 4)$ and the results compare to well-known properties of (G33).

The main idea for calculating the structure factor is based on taking advantage of socalled average unit cell (AUC). In order to get an AUC we have to: a) Choose an appropriate scattering vector $\mathbf{k}$, i.e. such a vector that $I(\mathbf{k}) \neq 0$. b) Place a reference grid on the direction along which we would like to calculate the AUC. This grid is periodical and its period equals to $\lambda=2 \pi / \mid \mathbf{k}$. c) Express positions $r$ of every atom as $r_{\mathrm{i}}=\alpha_{i} \cdot \lambda+u_{i} \alpha \in \mathrm{Z}$ d) The set of $u_{i}$ positions leads to the probability distribution of atoms' positions with reference to the grid i.e. the AUC. The Fourier transform of the AUC gives the structure factor for $\mathbf{k}$ and its higher harmonics. It can be easily shown that the AUC is just a projection of atomic surface (AS) along the perpendicular direction to $\mathbf{k}$. On the other hand we can get the AS lifting the AUC to higher-dimensions. For perfect structures these two approaches are fully equivalent. The AUC comes into its own when the structure becomes decorated or defected. Adding all imperfections to the structure factor which is based on the AUC is very easy. The AUC, unlike the 'cut-and-project method', is fully described only in physical space, so there is no ambiguity in doing this.

\section{Clusters}


There are many different kinds of clusters which we can use to cover the perfect, i.e. decorated only at vertices, Penrose lattice. We have already mentioned (K4), (K7), (K17) and (G33). In paper [7] the last two of them were discussed. They are, along with (K4) and (K7), presented in the figure 1. As you can see, clusters overlap. Overlapping reduces the number of independent types of atoms. Thus (G33) can be decorated only by 3 types of atoms (figure $2 \mathrm{a}$ ) and (K17) by seven types (figure $2 b$ ).

Here we should emphasize one thing. It is important to understand that by cluster we can mean either a set of atoms or some area. For example (G33) is a set of 33 atoms and the Cartwheel Decagon (CD), within which these 33 atoms lie, is an area located within the boundary of a decagon. We can then say that the CD is decorated by a set of atoms:(G33). The practical difference between $\mathrm{CD}$ and (G33) is that (G33) can cover only the atoms situated at the vertices of the perfect Penrose tiling and CD can cover the whole 2D surface. By (K4), (K7) and (K17) we mean kite-shaped areas decorated as it is shown in the figure 1. The area of $(K 7)$ is $\tau^{2}$-times larger than $(K 4)(\tau \approx 1.618)$. Similarly, the ratio of area of $(\mathrm{K} 17)$ to $(\mathrm{K} 7)$ is also $\tau^{2}$. The easiest way to cover Penrose tiling with (K4) is to find all thick rhombuses and exchanging them by (K4) cluster. All points of Penrose lattice belong to at least one thick rhombus. It is then possible to cover the whole Penrose lattice with (K4). In case of (K7) we should look for $\tau$-times larger thick rhombuses. The figure 3 depicts this procedure. Kite-cluster can be considered as a combination of thick and thin rhombus although the last one is halved along the shorter diagonal and merged to a thick rhombus along its edges. Because clusters overlap each other, the area they occupied is larger than the area of covered Penrose lattice. The ratio of these two areas is called 'degree of covering' (symbol: $\delta$ ) and it is discussed in detail in [7]. For instance for (G33) $\delta \approx 2.98$ and for (K17) $\delta \approx 1.53$.

For the perfect Penrose lattice atomic surface consists of four pentagons. Any part of the AS represents a probability distribution of certain set of atoms belonging to the Penrose lattice. For instance, as it was shown in [7], if we tried to find the probability distribution for any atom in a (K17) we would get a small, triangular area of AS. What would happen if we did this for every atom belonging to (K17) and additionally took into consideration every possible orientation of (K17)? Then we would get $10 \cdot 17=170$ overlapping triangular areas filling the whole AS. However, this filling isn't uniform. These 170 areas gather in 7 separated groups. They are depicted in the figure 5 and their vertices can be found in the table 1. The ratio of the area of the triangle representing the chosen group of atoms (multiplied by 10 different orientations) to the area of the atomic surface (about 17.205) gives the concentration of particular atom in the decorated Penrose tiling. A thick border in fig. 1 separates a chosen orientation from the other nine. We present only pentagons whose the third perpendicular coordinate $z$ is equal to 2 and 4 , because at $z=3$ there is a pentagon symmetrical to the one from $z=2$ and at $z=1$ there is a pentagon symmetrical to the one from $z=4$. Seven separated areas means seven independent atoms that can decorate (K17). Doing the same calculation for (K4) we would get 2 separated areas within AS i.e. two different atoms decorating Penrose lattice, for (K7): 4 independent atoms and for G33: 3 independent atoms.

As we can see the number of independent kinds of atoms and their relative concentrations are strongly dependent on the type of cluster. This knowledge can be crucial for the interpretation of experimental results. Knowing the real number of different atoms and their concentrations we can suggest the type of cluster which is the most adequate for refining an unknown structure from the experimental data.

Another interesting problem is whether it is possible to cover the whole Penrose lattice with a single type of rhombus. At first it seems rather possible. If we can cover the Penrose lattice with (K17) why not to try to extent this kite-shaped cluster into a large thick rhombus? The figure 4 depicts this issue. There are two thick rhombuses divided by dashed lines into 
(K17) and the additional part. As we can see this additional part differs in these two rhombuses. Both kinds of atoms and their positions are different. Positions marked with numbers 1 and 2 are the best example of the difference between these two rhombuses. All in all the final answer to this question seems to be negative. It is not possible to cover the whole Penrose lattice with only one type of rhombus.

\section{Structure factor}

For (K17)

the structure factor for diffraction peak indexed by $\left(n_{\mathrm{x}}, n_{\mathrm{y}}, m_{\mathrm{x}}, m_{\mathrm{y}}\right)$ at position $\mathbf{k}=\left(k_{\mathrm{x}}, k_{\mathrm{y}}\right)$ :

$$
k_{x}=k_{0 x}\left(n_{x}+\frac{m_{x}}{\tau}\right) ; \quad k_{y}=k_{0 y}\left(n_{y}+\frac{m_{y}}{\tau}\right)
$$

reads:

where $\quad \kappa=\left(\begin{array}{l}k_{x}-m_{x}(2 \tau-1) k_{0 x} \\ k_{y}-m_{y}(2 \tau-1) k_{0 y}\end{array}\right) \quad$ and $\quad \varphi_{1}=(\tau-1)\left(k_{x}+m_{x}(3-\tau) k_{0}\right)$

$\Gamma_{l j}$ parameter is a Fourier transform of the appropriate triangular distributions. Any decorating atom contributes to the structure factor by a linear combination of those parameters multiplied by the appropriate phase factor. Although the equation looks very complicated its application to particular decoration is straightforward. That allows us to use effectively the well-known fitting procedures (discussed separately on this conference by Kozakowski and Wolny). As the presented approach operates in physical space only it can be applied not only to arbitrary decorated clusters but also to some defected structure, including phasons and phonons.

For (K4) and (K7) this equations looks similarly. The sum is however over two or four independent atoms. $\Gamma_{l j}$ parameters differ as well. Now they are the Fourier transform of areas of AS representing atoms belonging to (K4) and (K7).

\section{Average Patterson analysis}

When refining a particular structure using the derived expression for the structure factor one should know the number of decorating atoms and estimate their positions in the cluster. The average Patterson function [11] is a very useful tool for that. This function is defined as an inverse Fourier transform of the measured diffraction pattern, additionally reduced to a single average unit cell. Average Patterson function is also an autocorrelation function of the probability distribution defined in statistical approach. Some points in the average Patterson map are strictly related to the reduced positions of decorating atoms.

The authors would like to acknowledge the State Committee of Scientific Research for financial support.

\section{References}

[1] N.G. de Bruijn, Proc. K. Ned. Akad. Wet. Ser. A 4339 (1981).

[2] M. Duneau, A. Katz, Phys. Rev. Lett. 542688 (1985). 
[3] P. Kramer and R. Neri, Acta Cryst. A 40580 (1984).

[4] V. Elser, Phys. Rev. B 314892 (1985).

[5] J. Wolny, Phil. Mag. A 77395 (1998).

[6] B. Kozakowski and J. Wolny, Structure factor for decorated Penrose tiling. http://arxiv.org/ftp/cond-mat/papers/0503/0503464.pdf, (2005).

[7] A. Dąbrowska, B. Kozakowski and J. Wolny, Acta Cryst. A 61350 (2005).

[8] P.J. Steinhardt P.J. and H.-C. Jeong, Nature 382433 (1996).

[9] P. Gummelt, Geometriae Dedicata 621 (1996).

[10] Coverings of Discrete Quasiperiodic Sets, Theory and Applications to Quasicrystals, edited by P. Kramer and Z. Papadopolos, Springer Tracts in Modern Physics, SpringerVerlag Berlin Heidelberg, 2003.

[11] J. Wolny and B. Kozakowski, Ferroelectricts 305217 (2004).

[12] B. Grueunbaum, G.C. Shephard, Tilings and Patterns, Freeman,New York, 1987, p. 562. 

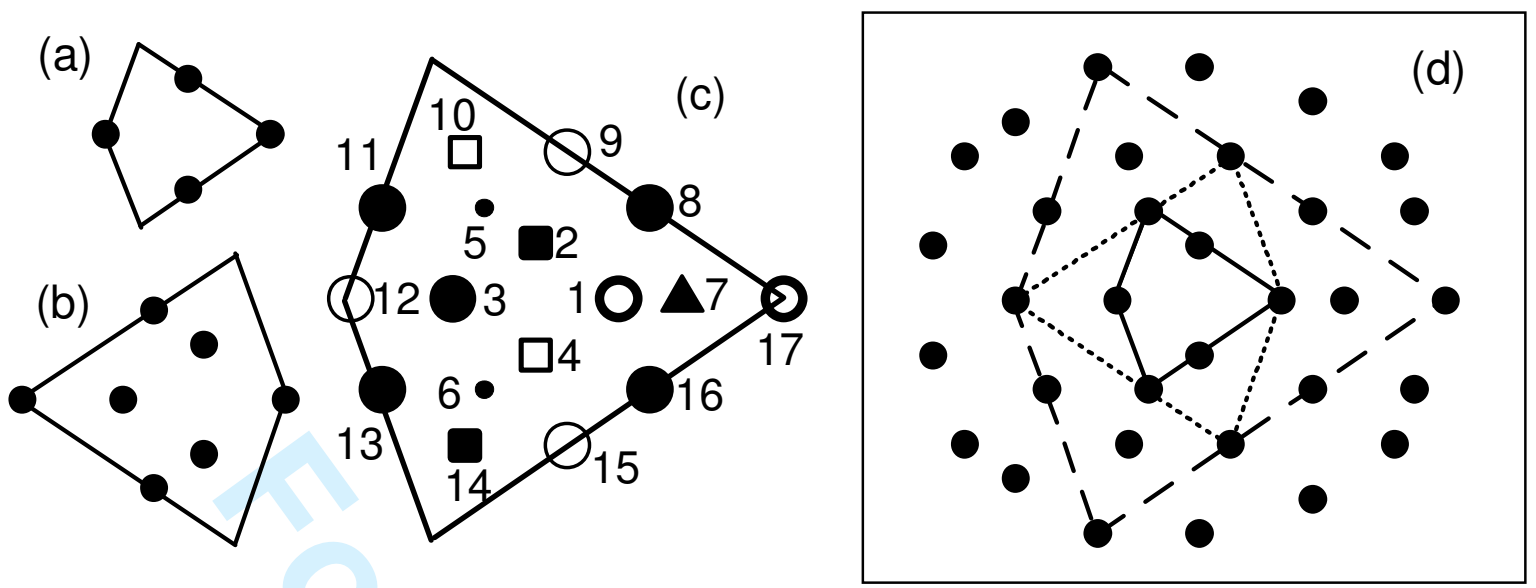


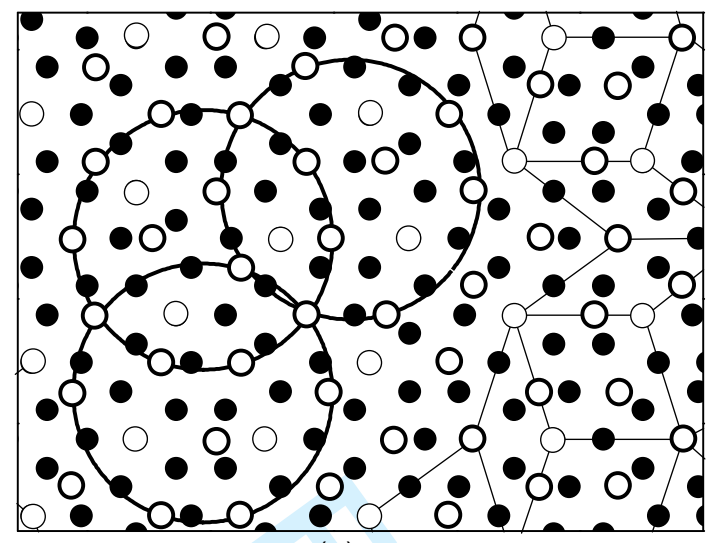

(a)

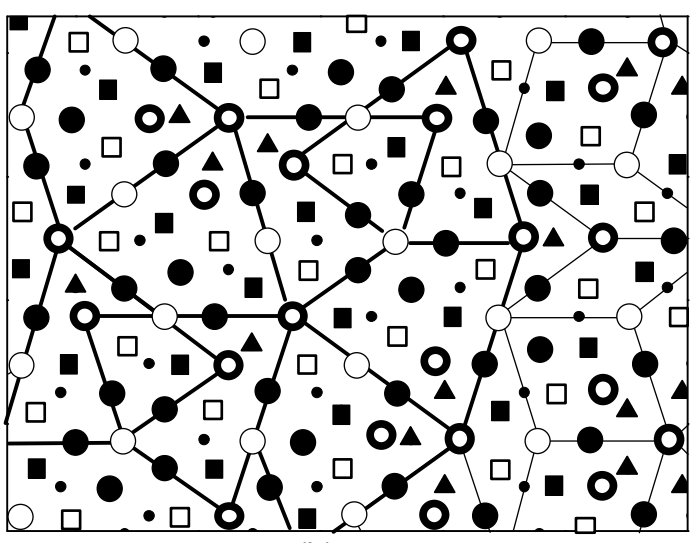

(b) 

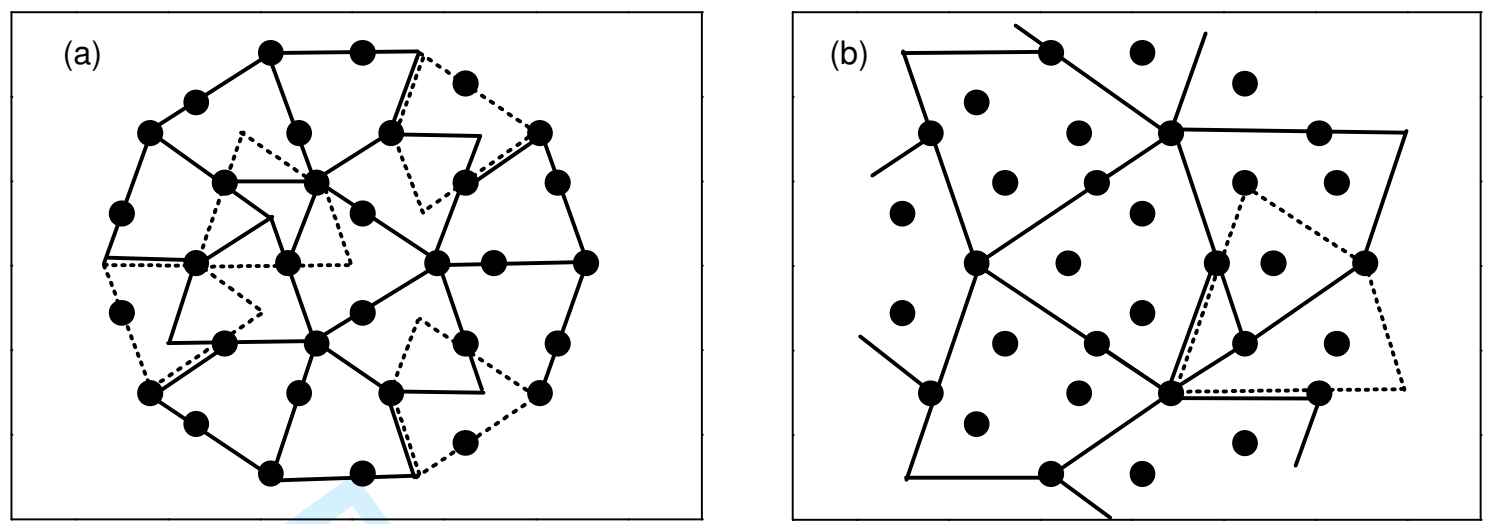


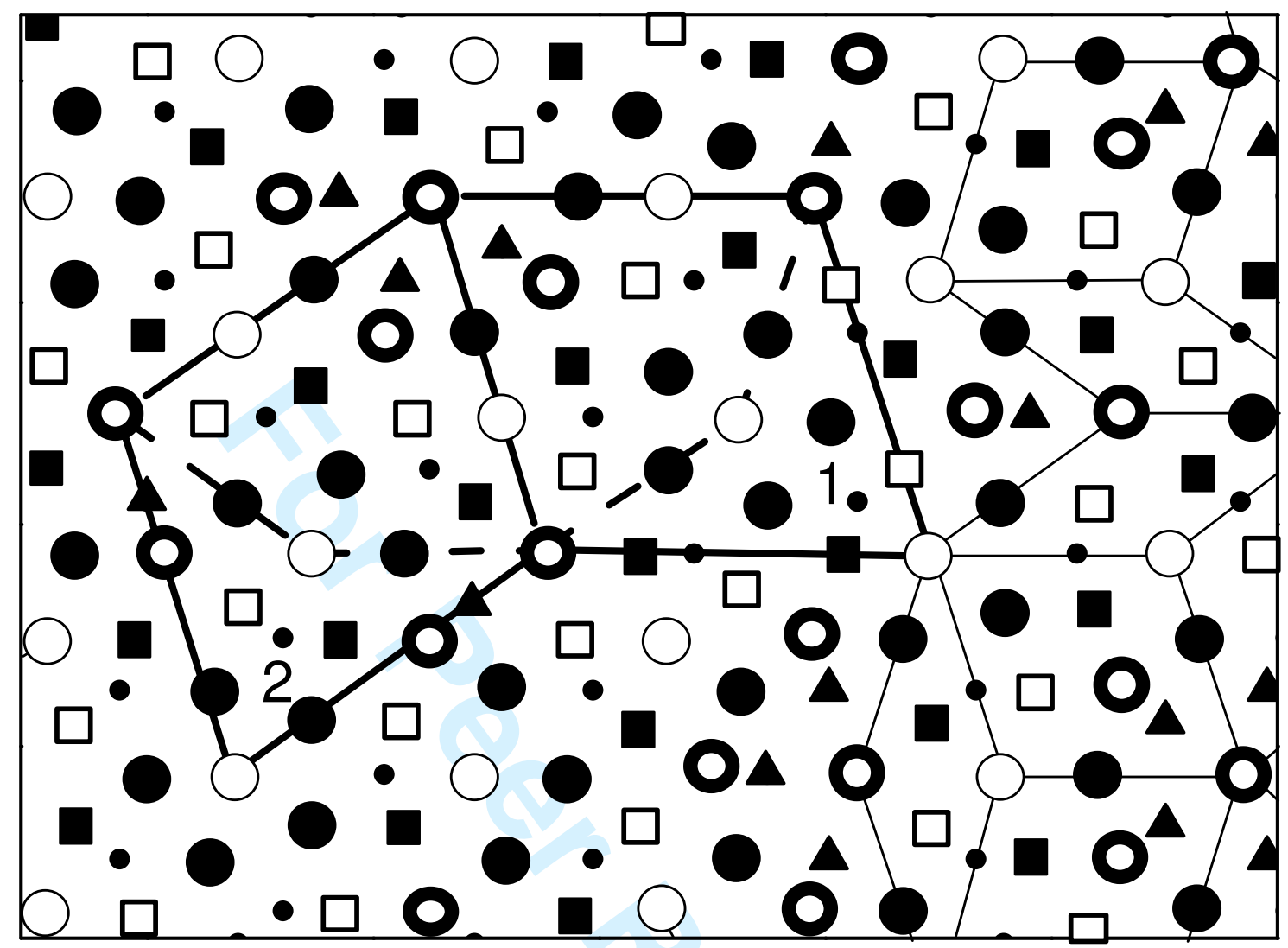


Page 9 of 10

Philosophical Magazine \& Philosophical Magazine Letters

1
2
3
4
5
6
7
8
9
9
10
11
12
13
14
15
16
17
18
19
20
21
22
23
24
25
26
26
27
28
29
30
31
32
33
3
34
35
36
37
38
39
40
41
42
4
4
4
4
5
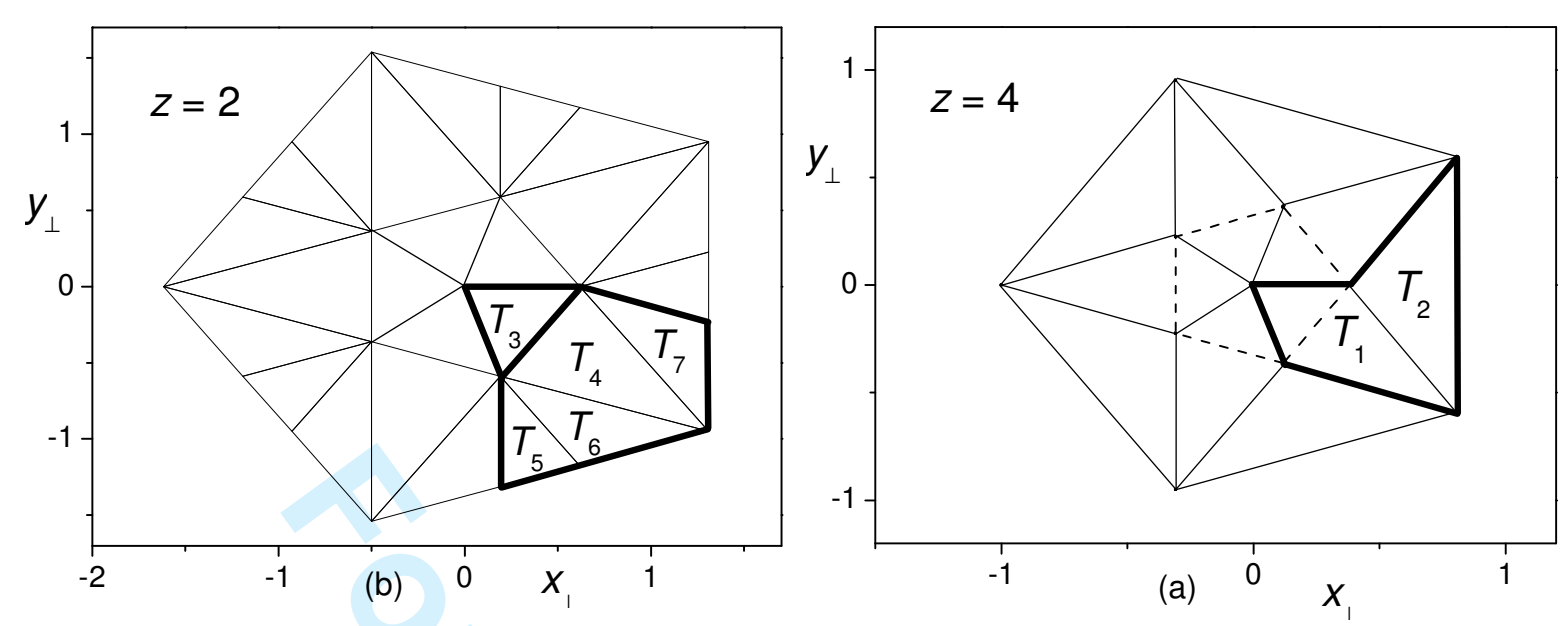

http://mc.manuscriptcentral.com/pm-pml 


\begin{tabular}{|c|c|c|c|c|c|c|c|}
\hline & Atomic numbers & Vertex 1 & Vertex 2 & Vertex 3 & Area & $C_{17}[\%]$ & $C_{33}[\%]$ \\
\hline$T_{1 \mathrm{~A}}$ & \multirow{2}{*}{1,17} & $\{0,0\}$ & $\{2(2-\tau), 0\}$ & $\{2 \tau-3, \tau-2\}$ & 0.069 & \multirow{2}{*}{13.0} & \multirow{3}{*}{27.6} \\
\hline$T_{1 \mathrm{~B}}$ & & $\{\tau, 1-\tau\}$ & $\{2(2-\tau), 0\}$ & $\{2 \tau-3, \tau-2\}$ & 0.155 & & \\
\hline$T_{2}$ & 5,6 & $\{2(2-\tau), 0\}$ & $\{\tau, \tau-1\}$ & $\{\tau, 1-\tau\}$ & 0.251 & 14.6 & \\
\hline$T_{3}$ & $9,12,15$ & $\{0,0\}$ & $\{2(\tau-1), 0\}$ & $\{2-\tau, 1-\tau\}$ & 0.182 & 10.6 & 10.6 \\
\hline$T_{4}$ & $3,8,11,13,16$ & $\{\tau+1,-1\}$ & $\{2(\tau-1), 0\}$ & $\{2-\tau, 1-\tau\}$ & 0.406 & 23.6 & \multirow{4}{*}{61.8} \\
\hline$T_{5}$ & 7 & $\{2-\tau, 1-\tau\}$ & $\{2-\tau, \tau-3\}$ & $\{2(\tau-1), 2(1-\tau)\}$ & 0.155 & 9.0 & \\
\hline$T_{6}$ & 2,14 & $\{2-\tau, 1-\tau\}$ & $\{\tau+1,-1\}$ & $\{2(\tau-1), 2(1-\tau)\}$ & 0.251 & 14.6 & \\
\hline$T_{7}$ & 4,10 & $\{2(\tau-1), 0\}$ & $\{\tau+1,3-2 \tau\}$ & $\{\tau+1,-1\}$ & 0.251 & 14.6 & \\
\hline
\end{tabular}

